\title{
Structural Analysis of a Vehicle Dynamics Model for Fault Detection and Isolation on the ROboMObil
}

\author{
Lok Man $\mathrm{Ho}^{1}$
}

\begin{abstract}
The ROboMObil, a mobility prototype under development at the DLR, differs from most current road vehicles with its high degree of overactuation with regards to the vehicle dynamics. This is due to the independent traction motor drives and steering actuators in each wheel, as well as one brake-by-wire actuator for each axle. Together with the large number of sensors, these provide opportunities for control and also lead to challenges for fault detection and isolation. In this study, the ROboMObil is represented by the dual track vehicle dynamics model. Structural analysis methods are used to determine the Minimally Structurally Overdetermined Sets of constraints. Each of these can be used to check the consistency of the constraints which it contains. A minimal set of MSO sets is selected to achieve the maximal isolation for the faults of interest. The validity of this method is illustrated using a simulation and simple analytic redundancy relations for selected MSO sets.
\end{abstract}

\section{INTRODUCTION}

In the context of fault diagnosis, structural analysis can be employed to determine redundancies that can be used for the detection and isolation of faults, before the numerical details of the constraints are fully available. The methods revolve around the determination of Minimally Structurally Overdetermined sets (MSO sets), which have a redundancy degree of one, meaning that each of them contain one more constraint than the number of variables. Several methods for finding all possible MSO sets of a system of constraints are compared in [1]. One method, discussed in [2], starts by determining a matching, in which one equation is assigned to each variable as the one used to calculate it. The unused equations are redundant and can then be used starting points to generate MSO sets. These can in turn be combined to generate the complete set of all MSO sets. Another method, presented in [3] and employed in this paper, removes one constraint from the full set at a time and checks for the degree of redundancy until a redundancy of one is reached. These methods have differing efficiencies depending on the number of constraints and the degree of redundancy of the full system.

The main goal of this work consists of the application of the aforementioned methods to the problem of fault detection and isolation (FDI) of the ROboMObil [4]. The ROboMObil is a prototype road vehicle with four independently controllable Wheel Robots, each comprising a steering actuator and an in-wheel traction motor drive. In addition there is also a brake-by-wire actuator in each axle. The vehicle

\footnotetext{
${ }^{1}$ L. Ho is with the German Aerospace Center (DLR), Institute of System Dynamics and Control, Oberpfaffenhofen, D-82334 Germany (phone: (+49)8153-28-3198; e-mail: lok.hoddlr.de).
}

is also equipped with sensors for the ego-motion of the vehicle. In order to systematically detect and isolate the component faults, structural analysis is used to search for the minimal set of MSO sets which achieves the maximal isolation properties. This is then used as a basis for creating a bank of residual generators for fault isolation.

In this paper, we employ the structural analysis within selected levels of a hierarchical FDI scheme. Hierarchical and distributed approaches to FDI of vehicle dynamics control systems with varying complexities are common, a systematic example can be found in [5]. Compared to a global FDI problem, the hierarchical approach has the advantage of reduction in the complexity of the individual FDI problems. A comparable problem is investigated in [6], in which fault detection filters for a Steer-by-Wire vehicle with individually steerable front wheels were developed. This paper aims to use structural analysis techniques to design the isolation matrix, a problem which becomes more difficult if relying on inspection-based methods for larger systems.

The paper is organized as follows. In Section II, the vehicle dynamics system to be analysed is described by its components, and also the scope of the analysis is defined. In Section III, a brief introduction to the structural analysis method is provided. Section IV describes the vehicle dynamics model used as well as the modelling of the faults. Section V demonstrates the application of the structural analysis methods to the model. To illustrate the results of this analysis, two sample faults are isolated using simple analytical redundancy relations (ARRs) based on the relevant MSO sets. The validity of the ARRs is shown using a MATLAB simulation of a dual-track model. A discussion of the results and further work can be found in Section VI.

\section{COMPONENT DESCRIPTION AND SCOPE OF ANALYSIS}

The hierarchy of system components in a Drive-by-Wire vehicle dynamics system is shown in Figure 1. In the analysis of this system, we consider both the levels of the vehicle dynamics systems and the intelligent actuators and sensors. The operation of the central vehicle dynamics control system (motion controller) is outside the scope of this study. To illustrate the boundaries between the intelligent actuators and the vehicle dynamics system, the actuators and their primary functions are listed below.

1) Traction motor drive actuator (TMD-A): closed-loop control of the motor current

2) Disc brake actuator (DB-A): closed-loop control of the hydraulic pressure, and the inner control loops of motor 
velocity and motor current

3) Steering actuator (S-A): closed-loop control of the steering angle measured using a sensor mounted on the steering axis, and the inner control loops of motor velocity and motor current

Each of the intelligent actuator subsystems is considered to provide a service which detects local faults. These local faults comprise those which prevent the demand from being realised with the normal behaviour. For an electromechanical actuator, this typically includes short-circuits, loss of power, other electrical faults, jammed transmission, sensor faults and control unit faults. A study of fault diagnosis of a steering actuator at this level can be found in [7].

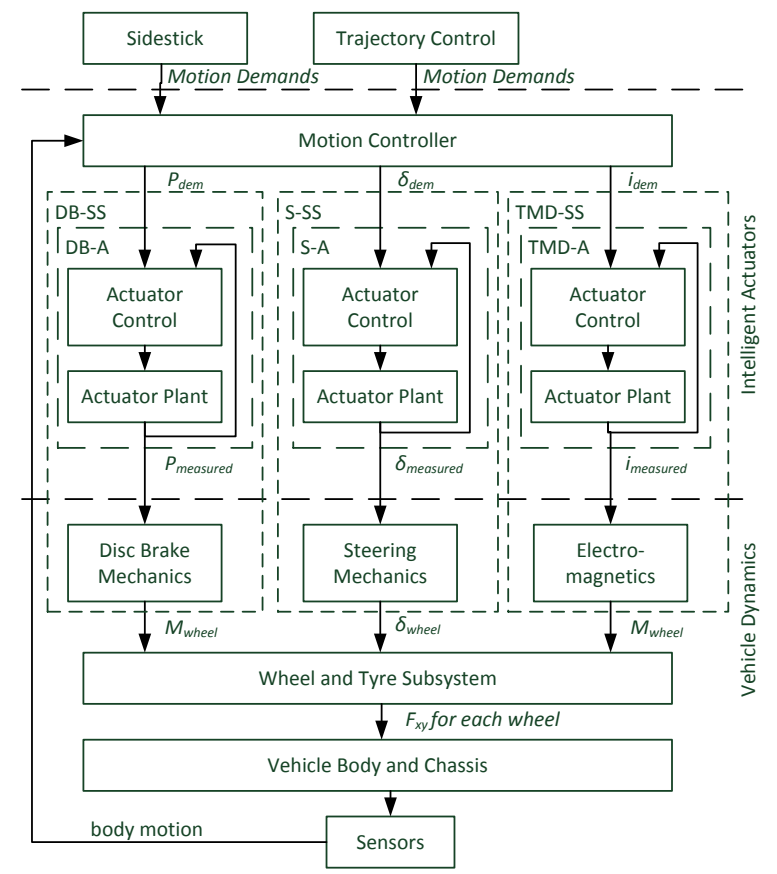

Fig. 1. Drive-by-Wire vehicle dynamics system components. Symbols used: $\mathrm{DB}=$ disc brake. $\mathrm{S}=$ steering. $\mathrm{TMD}=$ traction motor drive. $\mathrm{x}-\mathrm{SS}=$ subsystem. $\mathrm{x}-$ A=actuator. $M_{w h e e l}=$ wheel torque. $\delta_{w h e e l}=$ steering angle (vehicle coordinates). $P$ =pressure. $\delta=$ steering angle. dem=demand. $F_{x y}=$ horizontal vector force on road-tyre contact

We now define the functions and considered faults of the subsystems, each containing the intelligent actuators and the mechanism which generates the vehicle dynamics forces.

\section{A. Traction Motor Drive Subsystem}

The function of the traction motor drive subsystem is to generate a torque at the respective wheel. This subsystem contains the TMD-A (see Figure 1) on the intelligent actuator level. The fault considered at the vehicle dynamics level is a change in the relationship between the motor current, which is the output of the TMD-A, and the wheel torque. Possible causes for this type of fault include over-temperature and excessive friction in the bearings.

\section{B. Disc Brakes Subsystem (DB-SS)}

The function of the disc brake subsystem is to produce a wheel torque to slow down the vehicle. This subsystem contains the DB-A (see Figure 1) on the intelligent actuator level and the disc brake mechanics on the vehicle dynamics level. The fault considered in the disc brake mechanics is a change in the relationship between brake pressure and wheel braking torque, due to, for example damaged brake pads or blocked brake lines.

\section{Steering Subsystem (S-SS)}

The function of the steering subystem is to rotationally position the wheel carrier in vehicle coordinates to track the steering angle demand. This subsystem contains the SA (see Figure 1) on the intelligent actuator level, and the steering mechanics on the vehicle dynamics level. The S-A controls the steering angle in the local actuator coordinates. A possible fault in the steering mechanics could be due to bad calibration or incorrect mounting, such that there is an offset between the steering angle in vehicle coordinates and the angle measured by the sensor.

\section{Tyre and Wheel Subsystem}

The function of the tyre and wheel is to generate forces on the tyre-road interface. Faults occur when this relationship is not satisfied due to, e.g., low tyre pressure or a flat tyre.

\section{E. Sensors}

An ideal sensor provides an exact measurement of a system variable. A fault is thus defined as $y_{\text {measured }} \neq$ $y_{\text {actual }}$. Possible causes include sensor power loss, incorrect mounting and calibration, and sensor damage. Besides those sensors within the intelligent actuators, there are also ones which measure body motion (accelerations $a_{x}, a_{y}$, yaw rate $\dot{\psi}$ and velocities $v_{x}$ and $v_{y}$ ) as well as wheel speeds $\omega_{i}$.

\section{STRUCTURAL ANALYSIS}

The method for finding all the MSO sets presented in [3] was chosen for this work. A brief description of the method is described here, and the reader should refer to the original work for further details and proofs. The basic algorithm involves starting from the full system of constraints and removing them one at a time. At each step the set of the remaining constraints is checked for its degree of redundancy. When this is equal to one, then an MSO has been found. This algorithm is further optimised in [3] with steps 1-3. Step 4 is an additional step proposed by the author.

1) Keep track of the removed equations so that the same equations are not removed multiple times as different permutations

2) Use the Dulmage-Mendelsohn decomposition at each step to consider only the overconstrained part of the system

3) Simplify the model at each step by combining sets of constraints into equivalence sets where possible

4) Reduce the number of constraints and MSO sets by identifying "intermediate variables". These are variables that can be calculated from known variables via a "tree" of constraints that are not affected by faults. The replacement of this intermediate variable and the tree 
of constraints by a single known variable only removes low-priority MSO sets from the discovered collection of MSO sets. A low priority MSO set is one whose set of faults is exactly the union of the sets of faults affecting another group of MSO sets, and therefore does not extend the fault isolation properties of the set of MSO sets.

The topic of integral causality is raised in many publications regarding finding matchings [8], [9]. The use of integral causality in the equation solving sequence of an MSO requires that all the initial conditions are available, while the use of differential causality requires all necessary derivatives to be computable. In the currently considered task, derivatives can be well approximated given the low sample time, while initial conditions can be approximated using observers. Therefore both integral and differential causality are permitted.

Once all the MSO sets have been found, the faults which affect each of these MSO sets have to be determined. In the original structural model, faults are associated with constraints which they affect. The set of faults associated with an MSO set is the union of those associated with the contained constraints:

$$
F_{M S O_{i}}=\cup F_{j}, \forall j \mid c_{j} \subset M S O_{i}
$$

where $c_{j}$ is the $j$-th constraint, $F_{j}$ is the set of faults associated with $c_{j}$, and $M S O_{i}$ is the $i$-th MSO set. This information can be represented in the incidence matrix, which is a logical matrix where each row indicates the faults associated with that particular MSO set. This can be further processed to produce the fault matrix, which has fault numbers on both axes. A positive entry in the $i j$-th element indicates that the fault signature of the $i$-th fault can explain the occurrence of the $j$-th fault. Examples of the incidence and fault matrices can be seen in Figure 5 and Figure 4 respectively.

The subsets to be used for residual generation are selected by considering their contribution to fault isolation, as proposed in [10]. The MSO sets are first ranked by their usefulness for detecting the faults in the set. This has an inverse relationship with the uncertainty in the constraints of the MSO set, in that the influence of the faults on the residual must be greater than the effects due to the uncertainties for the faults to be detectable. A simplified measure of the uncertainty is the number of constraints in the MSO set, assuming that each contributes the same uncertainty. Starting from the first MSO set in this ordered list, each one is added to the selection if its addition to the already selected set of MSO sets leads to an increase in the fault isolation ability. A reduction of positive elements in the fault matrix indicates an increase in the fault detection ability. This is discussed in more detail in the example in Section V.

\section{MODELLING OF VEHICLE DYNAMICS AND FAULTS}

In this paper the structural analysis methods are applied to the dual track vehicle dynamics model. The model used here is based on the equations from [11], with simplifications to the vertical dynamics and tyre load calculations using the quasi-static assumption. The variables used in the original model are shown in Figure 2, while a block diagram of the model is shown in Figure 3. The original model calculates the vertical tyre loads by considering the vertical dynamics of each wheel with the spring and damper, as well as tyre stiffness. The quasi-static model simplifies this to a state-less calculation of the vertical tyre load based on the equations for steady-state cornering [12]. It can be reduced to this form:

$$
\left[\begin{array}{l}
F_{z 1} \\
F_{z 2} \\
F_{z 3} \\
F_{z 4}
\end{array}\right]^{\top}=\left[\begin{array}{ll}
\frac{m a_{x} h_{C o G}}{2 l} & \frac{m a_{y} h_{C o G}}{2 w\left(k_{f}+k_{r}\right)}
\end{array}\right]\left[\begin{array}{cc}
-1 & -k_{f} \\
-1 & k_{f} \\
1 & -k_{r} \\
1 & k_{r}
\end{array}\right]^{\top}
$$

$k_{f}$ and $k_{r}$ are constants which reflect the relative roll stiffness of the front and rear axles. This simplification significantly reduces the size of the model and speeds up the structural analysis using the current algorithms to an acceptable duration. The lost information about roll, pitch and the vertical wheel motion have a relatively minor effect on the actuator and sensor systems in this study, which primarily affect the planar vehicle dynamics.

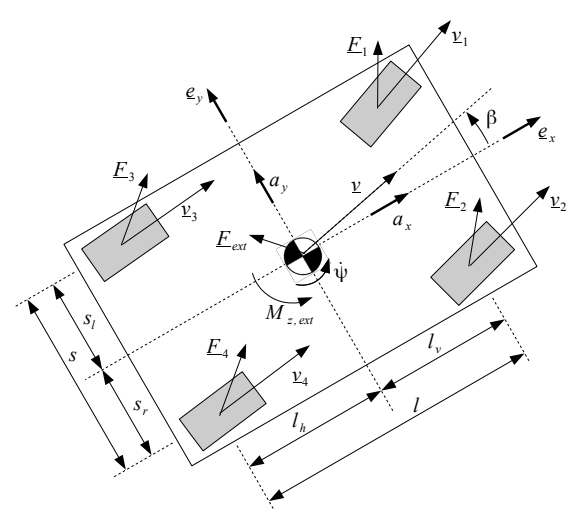

Fig. 2. Horizontal vehicle dynamics variables and parameters, reproduced according to [11].

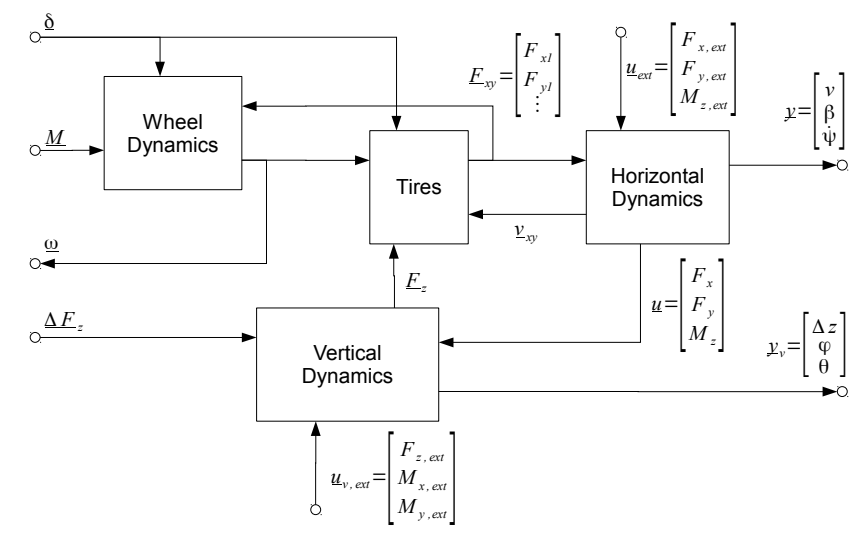

Fig. 3. Vehicle dynamics model block diagram in accordance to [11].

The faults considered in this analysis were described in the component analysis in Section II. They are represented 
as additive faults. The model equations for these faults are shown in TABLE I. In the table, the index $i$ indicates one instance for each of the 4 wheels, while the index $j$ indicates one instance for each of the two axles (front and rear).

TABLE I

LISTING OF FAULTS AND THEIR REPRESENTATIONS

\begin{tabular}{|c|c|c|}
\hline Fault & Equation & Fault \# \\
\hline $\begin{array}{l}\text { Acceleration sensor } \\
\text { fault }-\mathrm{x} \text { direction }\end{array}$ & $a_{m e a s_{-} x}=a_{x}+f_{a x}$ & $\mathrm{f} 1$ \\
\hline $\begin{array}{l}\text { Acceleration sensor } \\
\text { fault - y direction }\end{array}$ & $a_{m e a s_{-} y}=a_{y}+f_{a y}$ & $\mathrm{f} 2$ \\
\hline Yaw rate sensor fault & $\dot{\psi}_{\text {meas }}=\dot{\psi}+f_{\psi}$ & $\mathrm{f} 3$ \\
\hline $\begin{array}{l}\text { Velocity sensor fault - } \\
\mathrm{x} \text { direction }\end{array}$ & $v_{m e a s_{-} x}=v_{x}+f_{v x}$ & $\mathrm{f} 4$ \\
\hline $\begin{array}{l}\text { Velocity sensor fault - } \\
\text { y direction }\end{array}$ & $v_{m e a s_{-} y}=v_{y}+f_{v y}$ & f5 \\
\hline Steering angle offset & $\delta_{i}=\delta_{\text {meas_ } i}+f_{\delta i}$ & f6-f9 \\
\hline $\begin{array}{l}\text { Traction } \\
\text { torque } \\
\text { fault }\end{array}$ & $M_{T M i}=K_{T} i_{T M_{-} i}+f_{T M i}$ & f10-f13 \\
\hline $\begin{array}{l}\text { Friction brake torque } \\
\text { generation fault }\end{array}$ & $\begin{array}{l}M_{D B i}=\mu_{D B_{-} i} A_{D B_{-} i} P_{D B_{-} j}+ \\
f_{D B i}\end{array}$ & f14-f17 \\
\hline $\begin{array}{l}\text { Tyre force generation } \\
\text { fault }\end{array}$ & $\underline{F_{i}}=r_{F_{i}}\left(\underline{s_{i}}, F_{\max _{i}}\right)+f_{\text {tyre }_{-} i}$ & $\mathrm{f} 18-\mathrm{f} 21$ \\
\hline $\begin{array}{l}\text { Wheel speed sensor } \\
\text { fault }\end{array}$ & $\omega_{\text {meas } \_}=\omega_{i}+f_{\omega i}$ & f22-f25 \\
\hline
\end{tabular}

\section{ANALYSIS OF THE DUAL TRACK MODEL}

The algorithms for model simplification and MSO set search from [3] were programmed in MATLAB and then applied to the dual track model. Initially the full dual track model (with the detailed vertical dynamics) according to [11] was analysed. With this implementation of the model, the algorithm was found to be computationally intractable. This is the motivations for the use of the simplified dual track model described in Section IV.

The quasi-static vertical dynamics replaces 15 equations associated with vertical dynamics with 4 . This led to an equation system with 63 normal equations containing no known variables, 73 unknown variables and 19 measurements as known variables (with the same number of additional measurement equations). This leads to a structural redundancy of $\bar{\varphi}=63+19-73=9$. Note that the measurement equations shown in Table I were necessary to consider faults in the sensor measurements in this analysis framework. Groupings of equations related to measurements, slip calculation and tyre force dynamics were formed as per simplification step 4 in Section III, reducing the structure to 41 equations and 32 unknown variables.

\section{A. Finding the MSO Sets}

The search algorithm found 5161 unique MSO sets. It is generally possible to assign a physical interpretation to each of these sets. This was done in TABLE II for a selection of the MSO sets. In preparation for the next step of selecting a subset of the complete collection of MSO sets for fault isolation, some properties of these MSO sets are determined. These are:

- The number of variables in each MSO set

- The faults that affect each MSO set (see Figure 5). for the results for a selection of MSO sets)

The signature of a fault is the set of MSO sets which is sensitive to it. With the matrix visualisation shown in Figure 5 , the signature of fault $i$ can simply be determined by finding the filled elements in the $i$-th column.

\section{B. Fault Isolation}

This step determines the fault isolation properties using all the MSO sets, followed by the selection of a minimal subset of MSO sets which achieves the same fault isolation ability.

The fault matrix in Figure 4 demonstrates the isolation properties of the found MSO sets. The ordinate axis shows the fault that has occurred, and where the filled elements in that column indicate the faults that it could be interpreted as. Ideally only the diagonal elements are filled, meaning that each fault can be uniquely identified based on its fault signature.

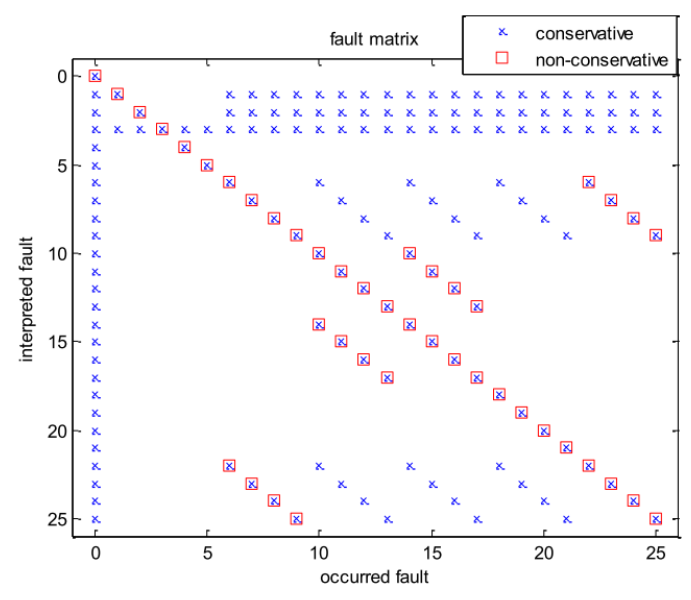

Fig. 4. Fault matrix

In Figure 4, the results of two methods of calculating the fault matrix are displayed. The conservative method (method A) is employed in [3] and does not consider the fulfilment of a consistency relation to be meaningful with regards to fault isolation. The explanation for this is that in real applications, often a consistency relation may be satisfied despite occurrence of faults that it contains, through certain non-zero combination of fault variables. Fault $i$ is said to "explain" fault $j$ if the signature of fault $i$ contains all the non-zero elements of fault $j$. The result is an asymmetric matrix shown by the ' $x$ ' in Figure 4. Note that "fault 0" additionally indicates the no-fault condition and is explained by every other fault according to this definition.

The alternative, less conservative method (method B) is to consider two faults as distinguishable from each other as long as their fault signatures are not identical. This produces a symmetrical fault matrix marked by the red squares in the 
figure. Note that even with this approach, perfect isolation of all the faults is not possible for the system being investigated.

\section{TABLE II}

MINIMAL SET OF MSO SETS SELECTED FOR ISOLATION

\begin{tabular}{|c|c|c|c|}
\hline MSO \# & \# vars & Interpretation & Faults numbers \\
\hline $1^{*}$ & 5 & $\begin{array}{l}\text { Kinematics. Measured } a_{x} \& \\
\text { measured velocities }\end{array}$ & $1,3,4,5$ \\
\hline $2 *$ & 5 & $\begin{array}{l}\text { Kinematics. Measured } a_{y} \& \\
\text { measured velocities }\end{array}$ & $2,3,4,5$ \\
\hline $3 *$ & 7 & $\begin{array}{l}\text { Kinematics. No } v_{y} \text { measure- } \\
\text { ment }\end{array}$ & $1,2,3,4$ \\
\hline $4 *$ & 7 & $\begin{array}{l}\text { Kinematics. No } v_{x} \text { measure- } \\
\text { ment }\end{array}$ & $1,2,3,5$ \\
\hline $15^{*}$ & 21 & $\begin{array}{l}\text { Wheel } 3 \text { dynamics, no use of } \\
\text { relations between } a_{x} \text { and } v_{x}\end{array}$ & $\begin{array}{l}1,2,3,4,5,8 \\
12,16,20,24\end{array}$ \\
\hline $21^{*}$ & 21 & $\begin{array}{l}\text { Wheel } 4 \text { dynamics, no use of } \\
\text { relations between } a_{x} \text { and } v_{x}\end{array}$ & $\begin{array}{l}1,2,3,4,5,9, \\
13,17,21,25\end{array}$ \\
\hline $5^{*}$ & 22 & $\begin{array}{l}\text { Wheel } 1 \text { dynamics, no mea- } \\
\text { surement of } v_{y}\end{array}$ & $\begin{array}{l}1,2,3,4,6,10 \\
14,18,22\end{array}$ \\
\hline $10 *$ & 22 & $\begin{array}{l}\text { Wheel } 2 \text { dynamics, no mea- } \\
\text { surement of } v_{y}\end{array}$ & $\begin{array}{l}1,2,3,4,7,11 \\
15,19,23\end{array}$ \\
\hline $27 *$ & 42 & $\begin{array}{l}\text { Full vehicle dynamics in the } \\
\text { x-direction using wheel speed } \\
\text { measurements and measured } \\
\text { road speed to derive slip and } \\
\text { wheel torques. }\end{array}$ & $\begin{array}{l}1,2,3,4,5,6,7 \\
8,9,18,19,20 \\
21,22,23,24,25\end{array}$ \\
\hline $30 *$ & 55 & $\begin{array}{l}\text { Full vehicle dynamics. Not } \\
\text { affected by: wheel } 2 \text { traction } \\
\text { and brake moment measure- } \\
\text { ments, rear-wheel (wheels } 3 \\
\text { and 4) tyre dynamics }\end{array}$ & $\begin{array}{l}1,2,3,5,6,7,8 \\
9,10,12,13,14, \\
16,17,18,19, \\
22,23,24,25\end{array}$ \\
\hline $36^{*}$ & 55 & $\begin{array}{l}\text { Full vehicle dynamics. Not } \\
\text { affected by: wheel } 2 \text { traction } \\
\text { and brake moment measure- } \\
\text { ments, wheels } 1 \text { and } 4 \text { tyre } \\
\text { dynamics }\end{array}$ & $\begin{array}{l}1,2,3,5,6,7,8, \\
9,10,12,13,14, \\
16,17,19,21, \\
22,23,24,25\end{array}$ \\
\hline $620 *$ & 55 & $\begin{array}{l}\text { Full vehicle dynamics. Not } \\
\text { affected by: wheel } 1 \text { and } 4 \\
\text { traction and brake moment } \\
\text { measurements, wheel } 2 \text { tyre } \\
\text { dynamics }\end{array}$ & $\begin{array}{l}1,2,3,4,5,6,7, \\
8,9,11,12,15, \\
16,18,20,21, \\
22,23,24,25\end{array}$ \\
\hline
\end{tabular}

The next step is the selection of the minimal subset of the MSO sets which provide the same isolation properties as the complete set. The MSO sets are first sorted in order of a cost function. In this case, the number of unknown variables in each MSO set is used. Starting from the one with the lowest cost, one MSO set is added to the selection at a time and the fault matrix is compared to that before the last addition. The MSO set is retained only if the isolation ability is improved, indicated by the deletion of at least one element in the matrix. The selection process was conducted for both evaluation methods of the fault matrix. In TABLE II, only the sets selected by method B are listed. The selection found using method B is a subset of that found using method $\mathrm{A}$ and will be used in the sequel.

The faults affecting each of these MSO sets are shown in Figure 5. Note that on the axis "MSO sets", the numbers do not correspond to the MSO set numbers on the table, but rather their index within the selection.

\section{Simulation Results}

In this section, the isolation of faults 4 and 5 is chosen as a demonstration example. We can observe that fault 4 and fault 5 have unique signatures in the combination of MSO sets 3 and 4 (row indices 3 and 4 in Figure 5). These signatures are $\{1,0\}$ and $\{0,1\}$ respectively. All other faults have signatures of either $\{0,0\}$ or $\{1,1\}$.



Fig. 5. Fault affecting each MSO set

The dual track model in [11] is simulated using parameters based on those of the ROboMObil. The vehicle motion is controlled by a geometric control algorithm, which assumes zero tyre slip when calculating the four steering angles and four combined wheel torques to produce the demanded vehicle motion 3-tuple of \{speed, side slip angle, yaw rate $\}$. This algorithm is described in [13].

MSO sets 3 and 4 involve the sets of constraints 1,2,3,4 and 1,2,3,5 respectively (see TABLE II). The involved constraints are listed below.

$$
\begin{gathered}
\text { Constraint\#1: } a_{x}=\dot{v}_{x}-v_{y} \psi \\
\text { Constraint\#2: } a_{y}=\dot{v}_{y}+v_{y} \psi \\
\text { Constraint\#3: } \dot{v}_{x}=\frac{d}{d t} v_{x} \\
\text { Constraint\#4: } \dot{v}_{y}=\frac{d}{d t} v_{y} \\
\text { Constraint\#5: } \dot{\psi}=\frac{d}{d t} \psi
\end{gathered}
$$

The ARR derived from MSO set 3 (ARR3) utilises the redundancies in the body acceleration and speed measurements, without using the lateral body velocity measurement $v_{\text {meas_y }}$. With simple algebraic manipulations and differentiations, the residual $r 3$ of ARR3 can be calculated by the following equations:

$$
\begin{gathered}
v_{x}=v_{\text {meas }_{x}}+d_{y} \dot{\psi} \text { meas } \\
p=\frac{d}{d t} v_{x}-a_{\text {meas_x }}
\end{gathered}
$$


$r 3=\dot{\psi}_{\text {meas }} \frac{d}{d t} p-p \frac{d}{d t} \dot{\psi}_{\text {meas }}-\left(a_{\text {meas }-y}-v_{x} \dot{\psi}_{\text {meas }}\right) \dot{\psi}_{\text {meas }}^{2}$

The ARR derived from MSO set 4 (ARR4) has the same structure with the coordinates $\mathrm{x}$ and $\mathrm{y}$ exchanged, with minor differences in sign. ARR4 does not use the measurement $v_{\text {meas } x}$. In the simulation shown in Figure 6, the ROboMObil is driven with a simple velocity profile with changing inputs on the lateral and rotational joystick axes. The middle and bottom plots show that ARR3 is sensitive only to $f_{v x}$ (fault 4), while ARR4 is only sensitive to $f_{v y}$ (fault 5). The residuals behave as designed to allow isolation of faults 4 and 5. The residual generation for many of the other MSO sets involve dealing with first order differential equations, which can be handled using the observer approach.
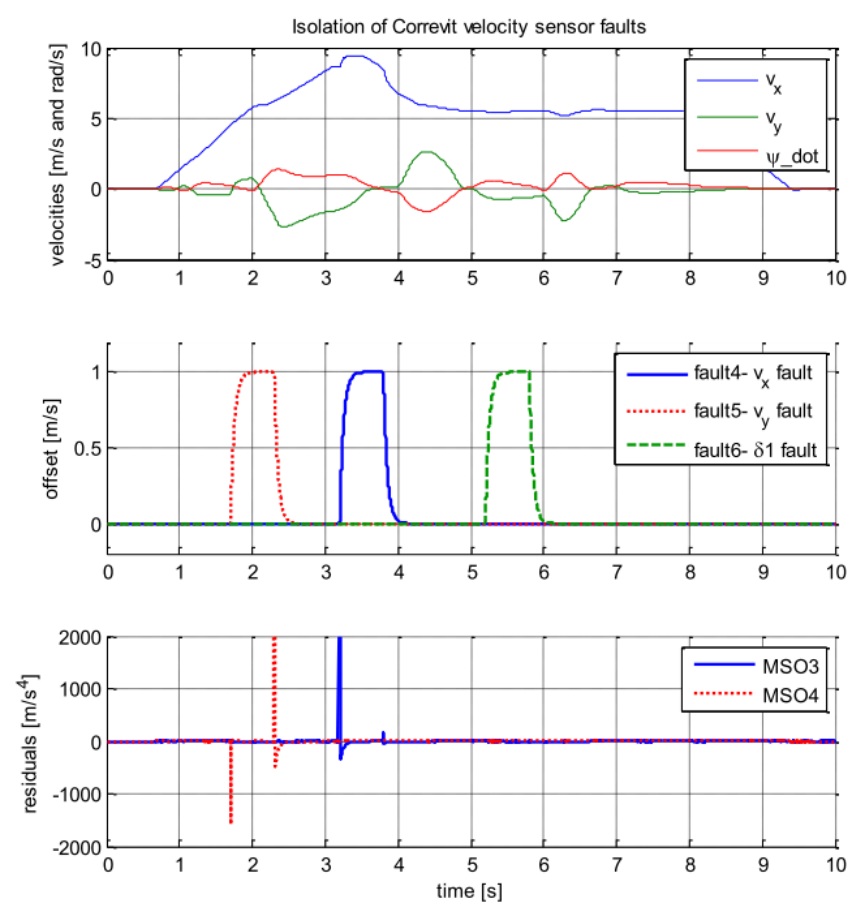

Fig. 6. Isolation of faults 4 and 5 ( $v_{x}$ and $v_{y}$ sensor faults respectively) using ARRs derived from MSO sets 3 and 4. We also see that fault 6 (fault with steering at wheel 1) has no effect on the two ARRs, as desired. The motion of the ROMO is generated using the following inputs to the virtual sidestick axes: custom longitudinal axis input (similar to profile of $v_{x}$ in the top plot), sawtooth lateral axis input, and a square pulse type signal for the additional side-slip angle input.

\section{DISCUSSION AND OUTLOOK}

The use of structural analysis for the systematic isolation of faults in the ROboMObil vehicle dynamics system has been demonstrated. Some points related to the structural analysis of this problem remain open. One concerns the verification of the residual generation using the MSO sets found using mixed causality. Another is the better estimation of uncertainty (due to parameter uncertainty and noise) in each MSO set during the selection of the minimal set of MSO sets for fault isolation.

Some sets of faults could not be decoupled using this analysis. Further courses of action are possible. One method, described in [3], involves considering some faults as unknowns and repeating the analysis starting with the search for MSO sets. A second method is to apply exact or approximate decoupling methods when developing residual generators.

The next step of this work following the structural analysis is the creation of a residual generator and evaluator for each MSO set. At this stage the model parameters, numerical equations, their uncertainties and the possibly unknown initial conditions must be considered. The improvement in the efficiency of the MSO search algorithm would enable its application to a broader system scope, possibly including the behaviours of the intelligent actuators and energy systems into a global analysis.

\section{ACKNOWLEDGMENT}

Lok Man Ho would like to thank Dr. Tilman Buente at the German Aerospace Center (DLR) for providing a MATLAB simulation of a dual-track model as well as the geometric vehicle control algorithm.

\section{REFERENCES}

[1] J. Armengol Llobet, T. Bregon, Anibal; Escobet, E. R. Gelso, M. Krysander, M. Nyberg, X. Olive, B. Pulido, and L. TraveMassuyes, "Minimal structurally overdetermined sets for residual generation: A comparison of alternative approaches," in 7th IFAC Symposium on Fault Detection, Supervision and Safety of Technical Processes, pp. 1480-1485, 2009.

[2] E. R. Gelso, S. M. Castillo, and J. Armengol, "An algorithm based on structural analysis for model-based fault diagnosis," in Proceedings of the 2008 conference on Artificial Intelligence Research and Development: Proceedings of the 11th International Conference of the Catalan Association for Artificial Intelligence, (Amsterdam, The Netherlands, The Netherlands), pp. 138-147, IOS Press, 2008.

[3] M. Krysander, J. Aslund, and M. Nyberg, "An efficient algorithm for finding minimal overconstrained subsystems for model-based diagnosis," Systems, Man and Cybernetics, Part A: Systems and Humans, IEEE Transactions on, vol. 38, pp. 197 -206, jan. 2008.

[4] J. Brembeck, L. Ho, A. Schaub, C. Satzger, J. Tobolar, J. Bals, and G. Hirzinger, "Romo - the robotic electric vehicle," in 22nd IAVSD International Symposium on Dynamics of Vehicle on Roads and Tracks, 14-19 August, 2011, August 2011.

[5] S. You and L. Jalics, "Hierarchical component-based fault diagnostics for by-wire systems," in SAE 2004 World Congress \& Exhibition, 2004. SAE Technical Paper 2004-01-0285,

[6] C. D. Gadda, Optimal fault-detection filter design for steer-by-wire vehicles. $\mathrm{PhD}$ thesis, Stanford University, 2008.

[7] J. S. Thomsen and M. Blanke, "Fault-tolerant actuator system for electrical steering of vehicles," in IEEE Industrial Electronics, IECON 2006 - 32nd Annual Conference on, (Paris, France), pp. 3597-3602, 6-10 Nov. 20062006.

[8] M. Blanke, M. Kinnaert, J. Lunze, and M. Staroswiecki, Diagnosis and Fault-Tolerant Control. 2nd ed., 2006.

[9] C. Svard and M. Nyberg, "Residual generators for fault diagnosis using computation sequences with mixed causality applied to automotive systems," Systems, Man and Cybernetics, Part A: Systems and Humans, IEEE Transactions on, vol. 40, pp. 1310 -1328, nov. 2010.

[10] M. Krysander and M. Nyberg, "Structural analysis for fault diagnosis of DAE systems utilizing graph theory and MSS sets," Tech. Rep. LiTH-R-2410, Department of Electrical Engineering, Linköpings Universitet, SE-581 83 Linköping, Sweden, 2002.

[11] R. Orend, Integrierte Fahrdynamikregelung mit Einzelradaktorik: ein Konzept zur Darstellung des fahrdynamischen Optimums. Berichte aus der Steuerungs- und Regelungstechnik, Shaker, 2006.

[12] T. D. Gillespie, Fundamentals of Vehicle Dynamics. SAE, 1 ed., 1992. ISBN: 978-1-56091-199-9.

[13] T. Buente, J. Brembeck, and L. M. Ho, "Human machine interface concept for interactive motion control of a highly maneuverable robotic vehicle," in Intelligent Vehicles Symposium (IV), 2011 IEEE, pp. 1170-1175, 2011. 